\title{
BMJ Open Randomised controlled trial comparing rituximab to mycophenolate mofetil in children and young adults with steroid- dependent idiopathic nephrotic syndrome: study protocol
}

\author{
Francesca Lugani, ${ }^{1}$ Andrea Angeletti (D) , ${ }^{1,2}$ Pietro Ravani, ${ }^{3}$ Marina Vivarelli, ${ }^{4}$ \\ Manuela Colucci, ${ }^{5}$ Gianluca Caridi, ${ }^{1}$ Enrico Verrina, ${ }^{2}$ Francesco Emma, ${ }^{4}$ \\ Gian Marco Ghiggeri ${ }^{1,2}$
}

To cite: Lugani F, Angeletti A, Ravani P, et al. Randomised controlled trial comparing rituximab to mycophenolate mofetil in children and young adults with steroid-dependent idiopathic nephrotic syndrome: study protocol. BMJ Open 2021;11:e052450. doi:10.1136/ bmjopen-2021-052450

- Prepublication history and additional supplemental material for this paper are available online. To view these files, please visit the journal online (http://dx.doi.org/10.1136/ bmjopen-2021-052450).

$\mathrm{FL}$ and $\mathrm{AA}$ contributed equally.

Received 15 April 2021 Accepted 21 September 2021

Check for updates

(C) Author(s) (or their employer(s)) 2021. Re-use permitted under CC BY-NC. No commercial re-use. See rights and permissions. Published by BMJ.

For numbered affiliations see end of article.

Correspondence to Dr Gian Marco Ghiggeri; gmarcoghiggeri@gaslini.org

\section{ABSTRACT}

Introduction Glucocorticoids induce remission in $90 \%$ of children with idiopathic nephrotic syndrome (INS). Some become steroid-dependent (SD) and require the addition of steroid sparing drugs such as calcineurin-inhibitors (CNI) or cyclophosphamide, to maintain remission. Considering the toxicity of these drugs, alternative interventions are needed for long-term treatment. The anti-CD20 antibody rituximab has shown promising steroid-sparing properties, with conflicting results in complicated forms of SDINS. Mycophenolate mofetil (MMF) resulted effective in maintaining free-steroid remission, however, studies are limited to few uncontrolled trials with reported different dose of MMF.

Methods and analysis This open-label, two-parallel-arm, superiority controlled randomised clinical trial will enrol children with SD-INS maintained in remission with oral glucocorticoids or CNI. Children and young adults will be randomised to either MMF $\left(1.200 \mathrm{mg} / \mathrm{m}^{2}\right)$ or rituximab $\left(375 \mathrm{mg} / \mathrm{m}^{2}\right)$ infusion. After enrolment, glucocorticoids will be tapered until complete withdrawal. We will enrol 160 children and young adults to detect as significant at the two-sided $p$ value of 0.01 with a power $>0.8 \mathrm{a}$ reduction in the risk of 1-year relapse (primary end-point). As secondary endpoints, we will compare the amount of glucocorticoids required to maintain complete remission at 6 and 24 months.

Ethics and dissemination The trial was approved by the local ethics boards (Comitato Etico Regione Liguria CER Liguria https://www.portalericerca-liguria.it/). We will publish the study results at international scientific meetings.

Trial registration numbers NCT004585152.

\section{INTRODUCTION}

Idiopathic nephrotic syndrome (INS) is a podocyte renal disease characterised by loss of the impermeability functions versus circulating proteins causing severe proteinuria and hypoalbuminaemia with oedema. In Western countries, NS affects 2-2.7 new
Strengths and limitations of this study

- Strengths include objective and clinical outcomes, identification of a well defined population, methods to reduce bias and careful collection of safety data.

- The possible crossing over, from one drug to the other, offers to patients more chances of clinical success.

- Limitations are the non-blinded interventions due to the different types of drugs administration and the primary end-point that is an intermediate rather than a final outcome.

children per 100.000 children per year and has a prevalence of 16 cases per 100.000. Oral corticosteroids are the cornerstone of therapy, inducing remission of NS in $\sim 90 \%$ of treated children. ${ }^{1}$ However, up to $85 \%$ of cases relapse within 5 years and many will develop steroid dependence NS (SDNS). ${ }^{2}$ According to Kidney Disease: Improving Global Outcomes (KDIGO) guidelines ${ }^{3}$ administration of low-dose prednisone is suggested to maintain remission in SD-INS (evidence 2C-D), and mycophenolate mofetil (MMF) or calcineurin inhibitors (CNI) as corticosteroid-sparing agents for children who develop serious corticosteroid-related adverse effects (evidence 1B). The list of steroid-sparing agents has minimally evolved over years: in the original 2012 guideline a single 6-month course of cyclophosphamide or chlorambucil were the first choice; 1-year levamisole in milder case and CNI and MMF for at least 12 months were alternatives. Rituximab was suggested only in those children who relapsed in spite of former alternatives. In the recent update of KDIGO, the order of presentation of steroid sparing agents to 
be used in SDNS is MMF, rituximab, cyclophosphamide and CNI that seems to have promoted rituximab as major option. ${ }^{4}$ Given the toxicity of the last two drugs there is a need to clarify which is, between MMF and rituximab, the most effective approach.

MMF inhibits purine synthesis in activated lymphocytes and reduces, in this way, the immune-response. A randomised controlled crossover trial involving 60 children with Frequntly Relapsing-INS compared the efficacy of MMF $\left(600 \mathrm{mg} / \mathrm{m}^{2}\right.$ two times a day) and cyclosporine directly. Relapses occurred in 36\% of subjects during MMF therapy vs only $15 \%$ during cyclosporine $(\mathrm{p}=0.06)$. The time without relapse was significantly longer with cyclosporine than with MMF during the first year $(\mathrm{p}<0.05)$, but not during the second year $(\mathrm{p}=0.36)$. Notably, adverse events were similar between the treatment arms with the exception of a lower estimated glomerular filtration rate (eGFR) and lower haemoglobin values in the cyclosporine arm suggesting more nephrotoxicity. ${ }^{5}$ Other small randomised controlled trials (RCTs) reported that MMF is inferior to CNI in both $\mathrm{SDNS}^{6}$ and in SRNS. ${ }^{7}$ There are still doubts on the dose to be administered, that is, few studies used $1 \mathrm{~g}$ two times a day but lower amounts could be considered. ${ }^{8}$ Moreover, the need to test the effective moiety mycophenolic acid exposure to optimise MMF therapy is still debated. ${ }^{9}$

Anti-CD20 chimeric and humanised antibodies induce apoptosis of B-cell and probably of other cells and also reduce the number of $\mathrm{B}$ memory cells that means the immunoglobulin secretory potential. Observational studies $^{1011}$ and two recent RCTs ${ }^{12}{ }^{13}$ support the use of rituximab in subjects SDNS, achieving stable steroid withdrawal formore then 1 year with a single infusion of rituximab $\left(375 \mathrm{mg} / \mathrm{m}^{2}\right)$. Suboptimal benefits were also obtained in complicated forms of the disease requiring the use of CNI. ${ }^{145}{ }^{15}$ recent meta-analysis ${ }^{16}$ concluded that rituximab is a valuable agent in SDNS but also suggested further comparative studies to address comparison with other drugs.

The aim of this trial is to test whether rituximab is superior to MMF in maintaining complete disease remission in children and young adults with steroid and/or CNIdependent NS and is a part of major study aimed to define the proper dose of MMF to be used in these cases. We initially planned to show superiority of rituximab (given in standard does of $375 \mathrm{mg} / \mathrm{m}^{2}$ ) compared with low dose MMF ( $350 \mathrm{mg} / \mathrm{m}^{2}$ two times a day) (NCT04402580): the trial was closed for futility after 30 subjects were enrolled because the $90 \%$ of subjects of the MMF arm relapsed after a median of 4.5 months. ${ }^{17}$ In the second part, we are seeking to compare superiority of rituximab (same dose) with MMF given in standard dose of $600 \mathrm{mg} / \mathrm{m}^{2}$ two times a day.

\section{METHODS AND DESIGN}

Most of the issues described below have been already presented in a companion paper describing another RCT comparing the effects of rituximab with ofatumumab in the same disease. ${ }^{18}$

\section{Rationale and justification of the active comparator}

The use of steroid-sparing agents is an important unmet clinical goal in paediatric nephrology, especially for children with complicated forms of SDNS requiring both glucocorticoids and CNI and new drugs represent an attractive opportunity. Due to its fully and strong affinity for the CD20 antigen, rituximab can be administered in a single dose, ${ }^{1319} 20$ much lower than previous reported multi-infusion protocols and with reduced risk of adverse events. ${ }^{21}$ Clinical trials in SDNS subjects have already demonstrated long-term effectiveness of single infusions, repeated over years if needing. ${ }^{12} 13$ MMF has been evaluated in previous studies ${ }^{5}$ and in small RCTs $^{67}$ showing effectiveness in reducing recurrence of proteinuria, but a standard dose is not defined. Head-to-head comparison of these two agents is justified on the basis of available data and awaited by clinicians who need drugs with reduced side effects for treating SDNS.

\section{Objectives}

This trial will test whether rituximab is able to achieve and maintain drug-free disease remission in subjects with SD-INS at 12 months (primary objective) and reduce the risk of relapse in a longer follow-up of 24 months (secondary objective) in children and young adults with SD-INS. This outcome will be compared with MMF, a drug recommended by Clinical Practice Guidelines (KDIGO) as steroid-sparing agents in the treatment of INS. This study will also collect information on occurrence of side effects as assessed by Common Terminology Criteria for Adverse Events (CTCAE) V.5.0 (ie, acute and long-term drug-related adverse events) and need to restart the use of glucocorticoids following the infusion of the trial interventions.

\section{Design}

This is an open-label, two-parallel-arm, controlled, phase II randomised clinical trial testing the superiority of rituximab over MMF in maintaining steroid-free and CNI-free disease remission in children with SD-INS (figure 1). Eligible participants are children and young adults (age between 3 and 24 years) with NS who are dependent on prednisone $0.3-1 \mathrm{mg} / \mathrm{kg} /$ day and have received prednisone for at least 6 months before enrolment. Previous treatment with MMF and CNI will be allowed. All participants, after inclusion criteria evaluation, will be engaged and after collection of the informed consent or assent, they will be randomised and start treatment. Randomisation will be stratified by age ( $<\mathrm{vs} \geq 9$ years) and centre. Each list will be generated using random numbers with blocks of variables size. Subjects randomised in the comparator arm will start, or continue, MMF $1200 \mathrm{mg} /$ $\mathrm{m}^{2}$ orally in two daily doses and will taper glucocorticoids after 15 days by $0.3 \mathrm{mg} / \mathrm{kg}$ per week until complete withdrawal. During the enrolment visit, instruction on 


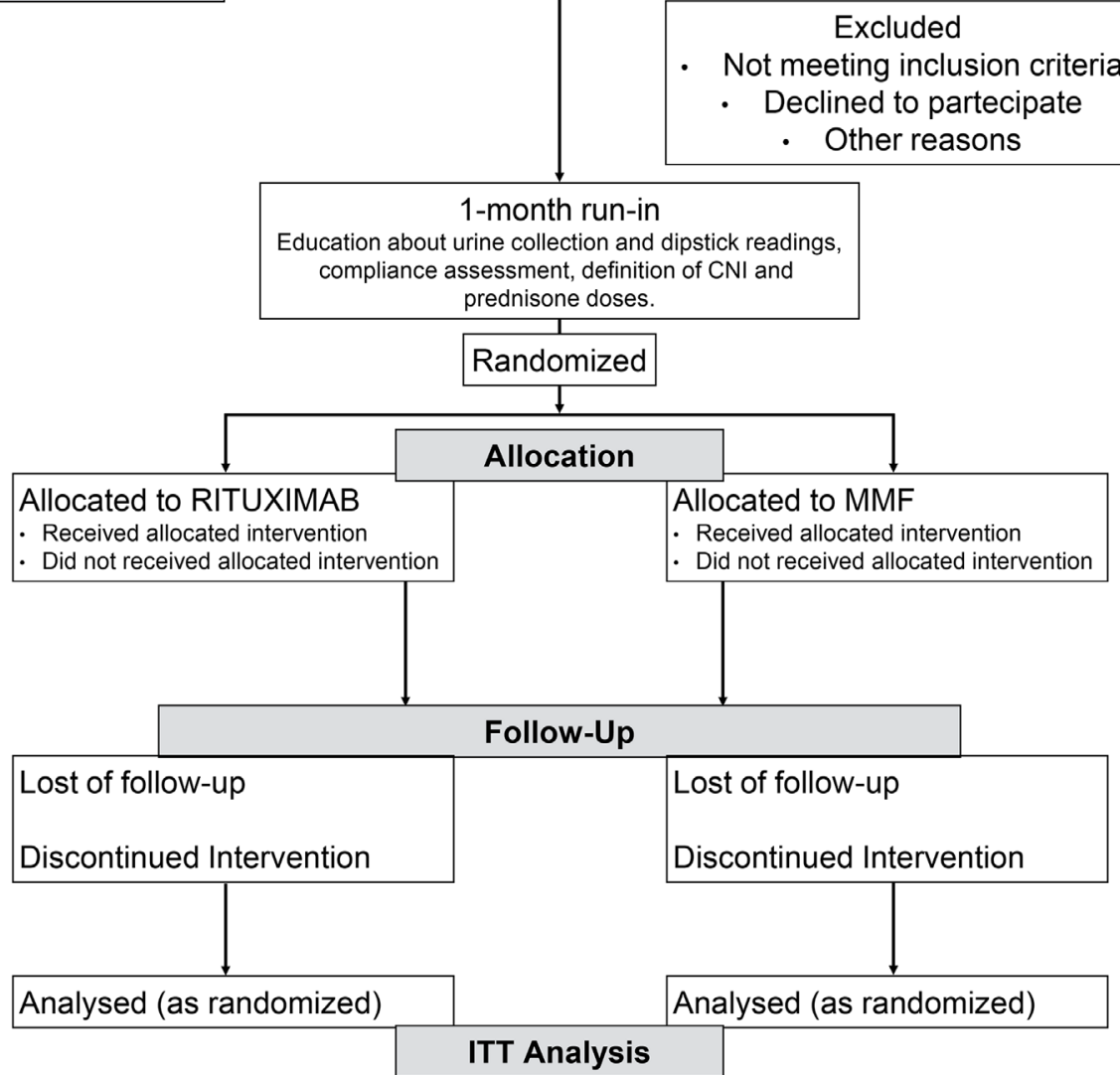

Figure 1 Schematic view of trial design. CNI, calcineurin inhibitors; ITT, intention to treat; MMF, mycophenolate mofetil.

urine collection and dipstick readings will be carefully reviewed and compliance assessed. Children or young adults randomised to the intervention arm will receive drug infusion (rituximab, $375 \mathrm{mg} / \mathrm{m}^{2}$ ). One month after infusion, other therapies already used before (as MMF or CNI) will be decreased by $50 \%$ and withdrawn within two additional weeks, similarly only MMF will be maintained in the comparator. All subjects will be followed for up to 24 months. In case of relapses during this period subjects will be treated with oral prednisone $(60 \mathrm{mg} / \mathrm{sqm}$ day $)$. Following remission, glucocorticoids will be maintained at the initial dose for 7 days and then tapered off by $0.3 \mathrm{mg}$ / $\mathrm{kg}$ per week until complete withdrawal in subjects of the MMF arm. Subjects of the intervention arm will instead be treated with another infusion of rituximab $(375 \mathrm{mg}$ / $\mathrm{m}^{2}$ ) immediately following steroid-induced remission. The study allows drop-in from one arm to the other after two relapses (ie, investigators will be allowed to use rituximab in the comparator arm and vice versa MMF in intervention arm). Study enrolment started in October 2020 and is expected to be completed within 1 year.

\section{Setting}

The trial will be carried out at two paediatric nephrology departments in Italy: the Nephrology Unit of the Giannina Gaslini Children's Hospital, Genoa and at the Nephrology Unit of the Bambino Gesù Pediatric Hospital, Rome. Interventions will be administered during an in-patient setting. Follow-up visits will be performed at the same institutions or by local nephrologist if travel to Genoa or Rome is not possible, in particular in case of a new COVID-19 pandemia waves.

\section{Eligibility criteria}

Inclusion criteria: Subjects aged 3-24 years, in complete disease remission and with a proven clinical history of SDNS are eligible for inclusion in the study. SDNS will be defined by two consecutive relapses during corticosteroid therapy tapering or within 14 days of steroid withdrawal. Subjects have been enrolled if they were receiving steroid alone or in association with MMF or CNI to maintain stable remission. Exclusion criteria were positivity to autoimmunity tests (antinuclear antibodies (ANA), antibodies anti-DNA (nDNA), anti-neutrophil cytoplasmic antibodies (ANCA)); reduction of C3 levels; eGFR $<90$ / $\mathrm{mL} / \mathrm{min} / 1.73 \mathrm{~m}^{2}$ evaluated according to revised Bedside Schwartz Formula for subjects between 2 and 17 years and with CKD-EPI Creatinine 2009 Equation for 18-24 years old subjects; pregnancy; neoplasm; previous or actual hepatitis B virus (HBV) (with hepatitis B surface antigen $(\mathrm{HBeAb})$ and antibody to hepatitis $\mathrm{B}$ core antigen (anti-HBc) Abs positivity) or hepatitis $\mathrm{C}$ virus (HCV) infection; CD20 B lymphocytes count $<2.5 \%$ and treatment with rituximab or cyclophosphamide in the last 6 months; previous reported severe adverse events (grade $\geq 3$ ) to study agents. 


\section{Participant identification}

Patients with SDNS afferring to the Nephrology units of Giannina Gaslini Children's Hospital, Genoa and of Bambino Gesù Pediatric Hospital, Rome will be interviewed to verify the existence of prerequisites for enrolment in the study. Information on treatments, randomisation procedure and follow-up steps will be given by specialised personnel of the medical staff; a sheet specifying the points above and the informed consent/ assent format will be given to patients or their parents depending to ages (not to children under 4 years). Before randomisation the assent will be required to children $<18$ years and the formal consent to their parents; patients over 18 years will be required to sign the consent. At this step, every patients will be assigned an unique number and the request of randomisation will be sent to a distant site analyst who is encharged of all the statistic procedures.

\section{Randomisation}

Participants will be randomised 1:1 to the intervention or active comparator arm. A distant site with no clinical involvement in the trial will generate two randomisation lists (for age $\geq 9$ years and $<9$ years) using permuted blocks of variable size. Stratification by age is motivated by the need to maximise the likelihood of balancing factors potentially affecting the effects of the intervention on outcomes, which are associated with age (disease duration, age at onset, relapse history and disease severity).${ }^{14} \mathrm{~A}$ study investigator responsible for recruitment will obtain signed consent (and assent for participants capable of assenting), assign a unique participant study number and request randomisation. An analyst from a distant site not involved in patient care, where the randomisation lists have been generated and kept concealed from the clinical investigators, will communicate the allocation arm to the study coordinator (based on the participant study number).

Randomisation 1:1 to rituximab (interventation) or to MMF (active comparator) will be generated by permuted block at a distant site by an analyst who is responsable of the final analysis of data. Patients in both arms will be stratified by age ( $\geq$ or $<9$ years) to balance potential modifiers of the drug response such as disease duration, other treatments and others. ${ }^{14}$ The allocation arm will be communicated by the principal investigator (PI) of the study to participants and to their parents depending on age.

\section{Treatment arms}

\section{Intervention}

Patients in the interventation arm will be treated with rituximab $375 \mathrm{mg} \mathrm{m}^{2}$ (Rixathon, Sandoz Schaftenau Biochemiestr. 106336 Langkampfen Austria) diluted in normal saline in accord to what already reported in this journal. ${ }^{18}$ Details on concentration of rituximab and speed of infusion have been already reported together with the composition of the premedication scheme. All the procedures will be carried out by a registered nurse.

\section{Active comparator}

Patients assigned to the acctive comparatot will be treated with MMF (Tillomed, Milano, I) $1.200 \mathrm{mg} \mathrm{m}^{2}$ in two doses.

Relevant concomitant care and interventions that are permitted or prohibited during the trial.

With the exception of those drugs listed as criteria for exclusion, starting from the signature of the informed consent, any other medication could be given based on the investigator decision who will be encharged to register all drugs in the report form. ACE inhibitors/angiotensin II receptor blockers (ARBs) will be stopped and replaced in hypertensive patients with other medications: carvedilol $0.2-1 \mathrm{mg} / \mathrm{kg}$ per day will be the first line choice followed by amlodipine $0.06-0.3 \mathrm{mg} / \mathrm{kg}$ per day; in all cases it will be associated restriction in dietary sodium intake.

\section{Outcomes}

Relapse of proteinuria or start of prednisone in the first year from treatment will be the primary end-point. The urinary protein/creatinine ratio (uPCr $>2.000 \mathrm{mg} / \mathrm{g}$ or $>200 \mathrm{mg} / \mathrm{mmol}$ ) is the basic criterium for defining relapse. Positive urine protein dipstick ( $>3+$ for 3 consecutive days) while being indicative of relapse will be confirmed by the uPCr ratio. Values of uPCr under the $50 \%$ of the limits above defines the partial remission. Failure to obtain complete withdraw of prednisone and/ or of MMF or CNI will indicate relapse.

Relapse of proteinuria after 24 months and number and severity of adverse events will be the secondary endpoints. Clinical aspects in the form of signs and symptoms, together with abnormal biochemical and haematology findings will define safety. They will be approached according to criteria as outlined in online supplemental appendix. Immune competence will be characterised and described as an ancillary aspect.

The follow-up will be closed at the completion of the study procedures at 12 (primary outcome) and 24 months (secondary outcome).

\section{Data collection, management, and analysis}

Data collection methods and adherence during follow-up

Out of relapses or acute events, visits will be scheduled at 1 month and every 3 months after treatments up to 24 months. During these visits, plasma proteins, immunoglobulins, lipids status and lymphocyte subpopulation characteristics will be determined (table 1). A 24-hour urine collections for protein determination is requested at any visit. Daily dipstick will be registered in a diary form that will be sent every 1-2 months to the PI.

\section{Data management}

The PI will design a person deputed to record clinical and biochemical parameters in an electronic data sheet. The PI and the person encharged of the data storage at the Giannina Gaslini will be responsible for a correct data management. 


\section{Statistical methods}

Patient characteristics in the two arms of the study will be summarised by common quantitative and qualitative methods. Logistic regression will be utilised for the analysis of the primary outcome and specifically for the calculation of the cumulative risk of relapse at 6 and 12 months considering a two-sided $\mathrm{p}<0.05$ as indicative of statistical significance. Survival methods will address the risk and the average time of relapse by treatment groups. ${ }^{22}$ An intention-to-treat approach will be used in all cases considering patients randomly aderent to the protocol. Replacement of missing data will reflect the worse case scenario that is success in the active comparator arm and failure in the interventation group.

\section{Sample size}

We will enrol 160 children and young adults in this study, and follow them for 2 years. Assuming a baseline risk of relapse at 1 year of 0.65 among children assigned to rituximab, this sample size will allow to detect as significant at the two-sided $\mathrm{p}$ value of 0.01 with a power $>0.8 \mathrm{a}$ reduction in the risk of 1-year relapse of at least 0.3 (ie, from 0.65 to 0.35 ; risk reduction by 0.46 ). This sample size accounts for a total proportion of drop-out and drop-in $<10 \%$.

\section{Patient and public involvement}

No patients were involved in the study's design.

\section{Study withdrawal}

Participants will be informed of the right of withdrawing at any time from the study without furnishing any reason and people of the medical staff give from now their personal assurance that this will not modify their conduct. On the other end, the PI has the possibility to withdraw a patient in case new health occurrences advise against participation to the study. Among any other, development of glucocorticoid resistance, worsening of renal function by $5 \%$ GFR every 6 months, malignancy, pregnancy, severe hypertension, proven hypersensitivity or allergy to drugs represent potential valid conditions necessitating withdrawl. Legal and administrative reasons may also suggest withdrawl. Both medical and non medical causes will be reported in the register.

The sponsor itself may stop temporarily or permanently the study based on safety, ethical or other reasons. In this case, the PI and the sponsor will rapidly inform the independent ethics committee.

Data safety and monitoring board, study monitoring and endpoint adjudication committee

Interim analyses or prespecified stopping rules are not planed. The data safety and monitoring board (DSMB) is scheduled when $50 \%$ of patients will be enrolled to decide about safety. DSMB is composed of experts in methodology and clinical nephrology and is under the responsibility of the sponsor. Monitoring of the study procedures (case report forms compilation, periodical visits, laboratory data) will be

Table 1 Participants timeline

\begin{tabular}{|c|c|c|c|c|c|c|c|c|c|c|}
\hline \multirow{3}{*}{ Time point } & \multirow{3}{*}{$\frac{\text { Enrolment }}{-1}$} & \multirow{3}{*}{$\begin{array}{l}\text { Allocation and } \\
\text { treatment } \\
0\end{array}$} & \multicolumn{8}{|c|}{ Study period } \\
\hline & & & \multicolumn{7}{|c|}{ Follow-up } & \multirow{2}{*}{$\begin{array}{l}\text { Close-out } \\
t_{24}\end{array}$} \\
\hline & & & $t_{1}$ & $\mathbf{t}_{3}$ & $\mathbf{t}_{6}$ & $t_{9}$ & $t_{12}$ & $t_{15}$ & $\mathbf{t}_{18}$ & \\
\hline Relevant medical history & $x$ & & & & & & & & & \\
\hline Eligibility screen & $X$ & & & & & & & & & \\
\hline $\begin{array}{l}\text { Instruction about immunosuppressive } \\
\text { drugs tapering }\end{array}$ & $x$ & $x$ & & & & & & & & \\
\hline Allocation & & $X$ & & & & & & & & \\
\hline \multicolumn{11}{|l|}{ Iterventions } \\
\hline Dosage on 24-hour urine collection & $x$ & $x$ & $\mathrm{X}$ & $\mathrm{X}$ & $X$ & $x$ & $x$ & $x$ & $x$ & $x$ \\
\hline Physical examination and vital signs & $x$ & $x$ & $x$ & $x$ & $x$ & $x$ & $x$ & $x$ & $x$ & $x$ \\
\hline Haematology and biochemistry* & $x$ & $x$ & $\mathrm{X}$ & $x$ & $X$ & $x$ & $x$ & $x$ & $x$ & $x$ \\
\hline Adverse events data records & & $x$ & $x$ & $x$ & $x$ & $x$ & $x$ & $x$ & $x$ & $x$ \\
\hline
\end{tabular}

*Haematology and biochemistry includes: complete blood count, kidney function, plasma proteins immunoglobulins, lipid status - cholesterol and triglycerides - albumin, lymphocyte subpopulations - for CD 20 lymphocytes B count.

MMF, mycophenolate mofetil. 
done periodically by the PI or by a delegate who will have access to the study material. Number and frequency of visits will be determined by the number of patients enrolled ( 1 visit every 40 patients). At the end, a final visit will control the whole collection of documents and data reports. Detailed analysis of each subject document will be done in a blind manner by a special committee that will established the end of the study.

\section{ETHICS APPROVAL, TRIAL STATUS AND DISSEMINATION}

Before initiation of the study, we obtained written approval of the protocol, Informed Consent/assent Form and any information presented to potential subjects from the local Independent Ethics Committee (Comitato Etico Regione Liguria). We also obtained approval from the Italian Drug Agency (Agenzia Italiana del Farmaco, AIFA). The trial is currently recruiting: study enrolment started on 15 October 2020.

The completed randomised clinical trial study will be summarised in a final report that accurately and completely presents the study objectives, methods, results, limitations of the study and interpretation of findings. The authors of this study protocol will inform the contributing investigators (primary healthcare providers of study participants) in advance about any plans to publish or present data from this RCT. Any publications and presentations of the results (abstract in journals or newspapers, oral presentations, etc), either in whole or in part, by investigators or their representatives will require presubmission review by the authors of this study protocol and all coauthors.

\section{DISCUSSION}

According to 2012 KDIGO Reccomandations, ${ }^{3}$ CNI and cyclophosphamide represent the treatment of choice in case of SDNS, causing an high risk of systemic toxicity due to the long term administration with both glucocorticoidsand/or standard steroid-sparing agents.

Cyclophosphamide, popular in early 1980s, presents serious limitations mostly because readministration over time is avoided for neoplastic complications, therefore, current management is limited. CNI result effective in SDNS, but the consequent direct and specific renal injury over years limits a prolonged use. ${ }^{23}$ Alternative treatments such as rituximab and MMF are considered as effective options in the 2019 updated revision of KDIGO. ${ }^{4}$

Recent RCTs ${ }^{1213}$ support the use of rituximab as an effective steroid-sparing agent in SDNS, mainly in subgroup of children presenting with moderate glucocorticoidsdependence, resulting really sensitive to the drug. ${ }^{13}$ On the other hand, effectiveness of rituximab may be suboptimal in SDNS subjects who remain dependent to glucocorticoidsafter the start of CNI, representing a category of subjects for whom more evidence relative to the efficacy of rituximab are needing. ${ }^{1424}$
MMF has been tested in retrospective studies and in small RCTs, showing a satisfactory efficacy in treatment of SDNS, also if with inferior power than CNI. ${ }^{6}$

A recent meta-analysis ${ }^{16}$ confirmed effectiveness of rituximab still recommending further studies comparing rituximab with drugs, such as MMF, causing limited side effects.

This is the first RCT comparing the risk of relapse in patients with SDNS treated by one of the two drugs. There are several strengths in the study that are mainly based on the choice of a well-defined category of patients with glucocorticoid dependent NS and on the definition of clear outcomes. Blinding, allocation system and definition of follow-up steps and evaluation of safety together with the expertise in research and clinical nephrology reinforce the quality of the study. Finally, the possible crossing over from one drug to the other offers to patients more chances of clinical success.

The design of this trial has also limitations. First, interventions are not blinded because rituximab and MMF require different types of administration. Second, the primary end-point is an intermediate rather than a final outcome. However, the lab-based measures adopted to define disease relapse are objective and more distant outcomes, including cardiovascular or infectious complications of NS, or progression of kidney disease to kidney failure, are rarely observed during childhood. A very large multinational trial would be necessary to study these hard end-points. On the other hand, relapse of NS is an important outcome for children and their families, often requiring travel to the nephrology centre in order to access urgent care. Third, this trial will compare the risk of relapse at 1 year based on the first event that occurs. Finally, according to the study model, participants are maintained in the assigned arm for follow-up studies up to presentation of two relapses, after then relapsing subjects are crossed over to the comparator (see above). In this way, comparison of the effect of each treatment on the risk of repeated relapses is not included in the present trial.

In summary, this study addresses an intervention question that is relevant to children and young adults with SDNS and their families. Results from this study may impact the management of SDNS in young children, providing efficacy and safety of these therapies justifying a widespread in specific renal clinical setting.

Improvement in quality of life, reduction in hospitalisation rates and use of healthcare resources are other important expected benefits.

\footnotetext{
Author affiliations

${ }^{1}$ Laboratory on Molecular Nephrology, Division of Nephrology, Dialysis, Transplantation, Istituto Giannina Gaslini Istituto di Ricovero e Cura a Carattere Scientifico, Genova, Italy

${ }^{2}$ Division of Nephrology, Dialysis and Transplantation, Istituto Giannina Gaslini Istituto Pediatrico di Ricovero e Cura a Carattere Scientifico, Genova, Italy ${ }^{3}$ Division of Nephrology, University of Calgary, Calgary, Alberta, Canada ${ }^{4}$ Division of Nephrology, Bambino Gesù Children's Hospital Istituto di Ricovero e Cura a Carattere Scientifico, Rome, Italy
} 
${ }^{5}$ Renal Diseases Research Unit, Genetics and Rare Diseases Division, Bambino Gesù Children's Hospital Istituto di Ricovero e Cura a Carattere Scientifico, Rome, Italy

Acknowledgements The Institute Giannina Gaslini (trial sponsor) will provide logistic and financial support to the trial through grants from the Italian Ministry of Health (Ministero della Salute, Ricerca Finalizzata PE-2016-02361576 and Ricerca Corrente Anno 2021). We aknowledge the 'Fondazione Malattie Renali del Bambino' for providing help and support to our patients and their family. The sponsor will appoint a study monitor. We thank the members of the DSMB (Giovanni Candiano $\mathrm{PhD}$ and Antonella Trivelli MD) and end-point adjudication committee (Drs. Fabrizio Ginevri and Enrico Verrina).

Contributors PR, MV, FE and GMG were involved in conception and trial design and in drafting of the article. FL, AA, MC, GC and EV reviewed/edited the manuscript.

Funding Giannina Gaslini Children's Hospital and Bambino Gesù Pediatric Hospital will provide both intervention and active comparator drugs (rituximab and mycophenolate mofetil respectively) and the infrastructure for the trial. The project was funded through grants by the Italian Ministry of health ('Ricerca Finalizzata PE-2016-02361576').

Disclaimer The study sponsor had no role in the study design and protocol development of this study. The sponsor will not have any role in the collection, analysis or interpretation of the data, or in the writing of report for publication. The researchers have complete independence from the sources of funding in all aspects of this study.

Competing interests None declared.

Patient consent for publication Consent obtained from parent(s)/guardian(s).

Provenance and peer review Not commissioned; externally peer reviewed.

Supplemental material This content has been supplied by the author(s). It has not been vetted by BMJ Publishing Group Limited (BMJ) and may not have been peer-reviewed. Any opinions or recommendations discussed are solely those of the author(s) and are not endorsed by BMJ. BMJ disclaims all liability and responsibility arising from any reliance placed on the content. Where the content includes any translated material, BMJ does not warrant the accuracy and reliability of the translations (including but not limited to local regulations, clinical guidelines, terminology, drug names and drug dosages), and is not responsible for any error and/or omissions arising from translation and adaptation or otherwise.

Open access This is an open access article distributed in accordance with the Creative Commons Attribution Non Commercial (CC BY-NC 4.0) license, which permits others to distribute, remix, adapt, build upon this work non-commercially, and license their derivative works on different terms, provided the original work is properly cited, appropriate credit is given, any changes made indicated, and the use is non-commercial. See: http://creativecommons.org/licenses/by-nc/4.0/.

\section{ORCID iD}

Andrea Angeletti http://orcid.org/0000-0002-6121-5326

\section{REFERENCES}

1 McEnery PT, Strife CF. Nephrotic syndrome in childhood. management and treatment in patients with minimal change disease, mesangial proliferation, or focal glomerulosclerosis. Pediatr Clin North Am 1982;29:875-94.

2 Kyrieleis HAC, Levtchenko EN, Wetzels JFM. Long-Term outcome after cyclophosphamide treatment in children with steroid-dependent and frequently relapsing minimal change nephrotic syndrome. Am J Kidney Dis 2007;49:592-7.

3 Radhakrishnan J, Cattran DC. The KDIGO practice guideline on glomerulonephritis: reading between the (guide)lines--application to the individual patient. Kidney Int 2012;82:840-56.

4 Floege J, Barbour SJ, Cattran DC, et al. Management and treatment of glomerular diseases (Part 1): conclusions from a kidney disease: improving global outcomes (KDIGO) controversies conference. Kidney Int 2019;95:268-80.
5 Gellermann J, Weber L, Pape L, et al. Mycophenolate mofetil versus cyclosporin $\mathrm{A}$ in children with frequently relapsing nephrotic syndrome. J Am Soc Nephrol 2013;24:1689-97.

6 Sinha A, Gupta A, Kalaivani M, et al. Mycophenolate mofetil is inferior to tacrolimus in sustaining remission in children with idiopathic steroid-resistant nephrotic syndrome. Kidney Int 2017:92:248-57.

7 Sinha A, Puraswani M, Kalaivani M, et al. Efficacy and safety of mycophenolate mofetil versus levamisole in frequently relapsing nephrotic syndrome: an open-label randomized controlled trial. Kidney Int 2019;95:210-8.

8 Moudgil A, Bagga A, Jordan SC. Mycophenolate mofetil therapy in frequently relapsing steroid-dependent and steroid-resistant nephrotic syndrome of childhood: current status and future directions. Pediatr Nephrol 2005;20:1376-81.

9 Benz MR, Ehren R, Kleinert D, et al. Generation and validation of a limited sampling strategy to monitor mycophenolic acid exposure in children with nephrotic syndrome. Ther Drug Monit 2019;41:696-702.

10 Guigonis V, Dallocchio A, Baudouin V, et al. Rituximab treatment for severe steroid- or cyclosporine-dependent nephrotic syndrome: a multicentric series of 22 cases. Pediatr Nephrol 2008;23:1269-79.

11 Kemper MJ, Gellermann J, Habbig S, et al. Long-Term follow-up after rituximab for steroid-dependent idiopathic nephrotic syndrome. Nephrol Dial Transplant 2012;27:1910-5.

12 Ravani P, Rossi R, Bonanni A, et al. Rituximab in children with steroid-dependent nephrotic syndrome: a multicenter, open-label, Noninferiority, randomized controlled trial. J Am Soc Nephrol 2015;26:2259-66.

13 Ravani P, Lugani F, Pisani I, et al. Rituximab for very low dose steroiddependent nephrotic syndrome in children: a randomized controlled study. Pediatr Nephrol 2020;35:1437-44.

14 Ravani P, Magnasco A, Edefonti A, et al. Short-Term effects of rituximab in children with steroid- and calcineurin-dependent nephrotic syndrome: a randomized controlled trial. Clin J Am Soc Nephrol 2011;6:1308-15.

15 lijima K, Sako M, Nozu K, et al. Rituximab for childhood-onset, complicated, frequently relapsing nephrotic syndrome or steroiddependent nephrotic syndrome: a multicentre, double-blind, randomised, placebo-controlled trial. Lancet 2014;384:1273-81.

16 Larkins NG, Liu ID, Willis NS, et al. Non-corticosteroid immunosuppressive medications for steroid-sensitive nephrotic syndrome in children. Cochrane Database Syst Rev 2020;4:CD002290.

17 Ravani P, Lugani F, Drovandi S, et al. Rituximab vs low-dose mycophenolate mofetil in recurrence of steroid-dependent nephrotic syndrome in children and young adults. JAMA Pediatr 2021;175:631.

18 Ravani P, Bonanni A, Ghiggeri GM. Randomised controlled trial comparing ofatumumab to rituximab in children with steroiddependent and calcineurin inhibitor-dependent idiopathic nephrotic syndrome: study protocol. BMJ Open 2017;7:e013319.

19 Kamei K, Ito S, Nozu K, et al. Single dose of rituximab for refractory steroid-dependent nephrotic syndrome in children. Pediatr Nephrol 2009;24:1321-8.

20 Fujinaga S, Hirano D, Nishizaki N, et al. Single infusion of rituximab for persistent steroid-dependent minimal-change nephrotic syndrome after long-term cyclosporine. Pediatr Nephrol 2010;25:539-44.

21 Bonanni A, Calatroni M, D'Alessandro M, Signa S, et al. Adverse events linked with the use of chimeric and humanized anti-CD20 antibodies in children with idiopathic nephrotic syndrome. Br J Clin Pharmacol 2018;84:1238-49.

22 Ravani P, Ponticelli A, Siciliano C, et al. Rituximab is a safe and effective long-term treatment for children with steroid and calcineurin inhibitor-dependent idiopathic nephrotic syndrome. Kidney Int 2013;84:1025-33.

23 Magnasco A, Rossi A, Catarsi P, et al. Cyclosporin and organ specific toxicity: clinical aspects, pharmacogenetics and perspectives. Curr Clin Pharmacol 2008;3:166-73.

24 Basu B, Sander A, Roy B, et al. Efficacy of rituximab vs tacrolimus in pediatric corticosteroid-dependent nephrotic syndrome: a randomized clinical trial. JAMA Pediatr 2018;172:757-64. 